\title{
Para-oesophageal hernia of the stomach complicating Heller's operation
}

\author{
CYRIL HAVARD \\ From Bridgend General Hospital, Glamorgan
}

The soundness of Heller's operation for achalasia of the cardia has been stressed by Barrett and Franklin (1949), when they were surveying the unfavourable results of other operative procedures for this condition. They hold that the unfavourable results of these alternative operations are due mainly to the incompetence of the cardia which ensues, and point out that Heller's operation does nothing to interfere with the competence of the oesophago-gastric junction.

Not all patients treated for achalasia of the cardia by Heller's operation are completely cured symptomatically, however, and Ravitch (1958) suggests that these poor results might be due to a hiatus hernia, either pre-existing or post-operative. Demonstration of reflux up the oesophagus on radiographic examination of all unsatisfactory cases in a series of patients treated by Heller's operation is reported by Frobese, Stein, and Hawthorne (1961), and these authors go on to describe four cases of hiatus hernia after Heller's operation.

A further case of hiatus hernia is described here, occurring as a serious complication in the immediate post-operative period.

\section{CASE REPORT}

G. E., a 36-year-old single woman, was admitted to Bridgend General Hospital on 14 November 1960. She complained of increasing difficulty in swallowing for two years, the symptoms having been severe for six months. For the previous three months she had been able to swallow fluids only and she had lost $28 \mathrm{lb}$. $(12.7 \mathrm{~kg}$.) in weight. During the first year of her two-year history she had complained of heartburn, particularly in the morning, but there had been no recurrence of this during the second year. Regurgitant vomiting had been present for six months and had been severe for three months. On examination there were no abnormal physical signs apart from obvious loss of weight.

Radiography on 28 October 1960 had shown a grossly distended and elongated oesophagus due to achalasia of the cardia (Fig. 1).
On 16 November 1960 a left thoracotomy was performed, resecting the eighth rib, and the diagnosis of achalasia of the cardia confirmed. The muscle coats of the oesophagus were divided longitudinally over approximately $5 \mathrm{~cm}$. anteriorly, and the diaphragmatic hiatus was enlarged by blunt dissection to allow the muscle division to be extended an equal distance on to the stomach wall. The hiatus was repaired with interrupted catgut sutures. In the dissection a small opening was accidentally made in the ossophageal mucosa and this was also repaired with interrupted catgut sutures.

The chest was closed with basal and apical drainage tubes connected to underwater seals. Gentle suction of $5 \mathrm{~cm} . \mathrm{Hg}$ was applied to the drainage tubes while the patient was in the theatre recovery room and a radiographic examination later that day showed the left lung to be fully re-expanded.

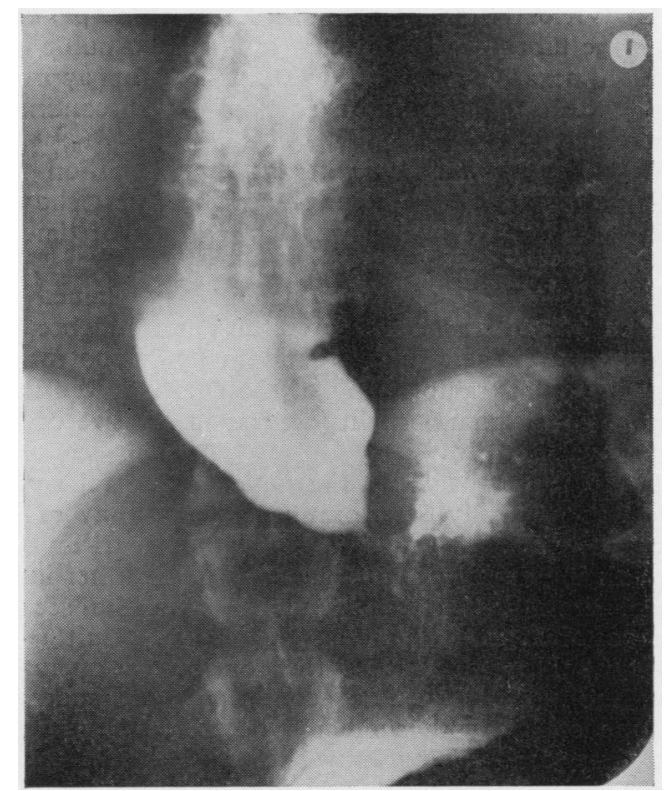

FIG. 1. Pre-operative radiograph after barium swallow showing the distended and elongated oesophagus with no evidence of a hiatus hernia. 


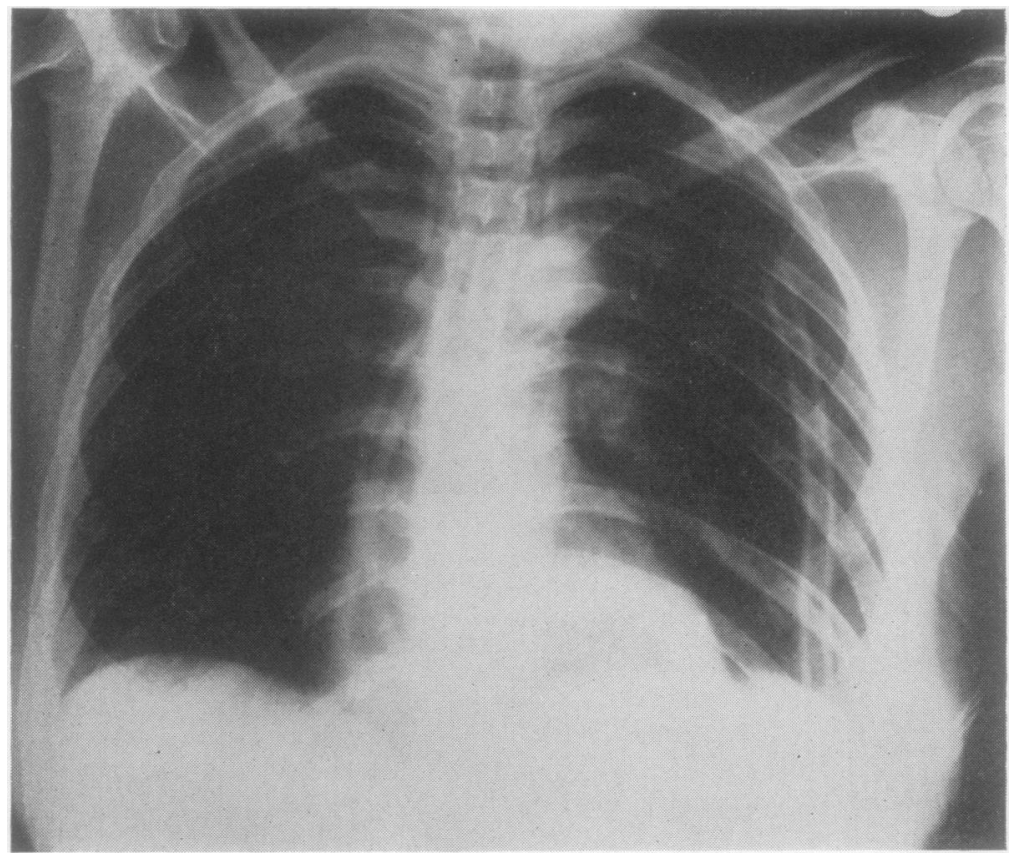

FIG. 2. A postero-anterior radiograph of the chest on the first post-operative day showiug a small fluid level.

Inadvertently, on returning to the ward the patient's drainage tubes were connected to a suction pump and a high negative pressure was applied for approximately three hours before suction was discontinued completely.

On 17 November 1960 her general condition was satisfactory though radiography of the chest showed a small fluid level which was thought at that time to be in a small pleural space (Fig. 2).

The following day the patient complained of sudden pain in the left side of the chest and became slightly dyspnoeic and temporarily hypotensive, though further radiography of the chest showed little change apart from a slight increase in the size of the fluid level.

By 19 November 1960 the patient's general condition was gradually deteriorating. Radiography of the chest (Fig. 3) then showed a large fluid level and, as the existing drain was not functioning, a further intercostal drain was inserted anteriorly in the second intercostal space without any significant result.

On 20 November 1960 , the fourth post-operative day, the patient's general condition very suddenly became critical with sudden hypotension, semicoma, and dyspnoea. Radiography showed a second apical fluid level with gross atelectasis of the left lung and pleural effusion (Fig. 4).

With a diagnosis of oesophago-pleural fistula and tension pneumothorax the left pleura was re-explored on 20 November 1960. After aspirating a large quantity of brown watery fluid, the findings were as follows. Two-thirds of the stomach had herniated through the hiatus into the pleural cavity alongside the oesophagus. The margins of the hiatus had formed a tight band around the stomach, causing linear gangrenous patches in the stomach wall and resulting in a high intestinal obstruction. The opening in the oesophageal mucosa made at the time of the Heller's operation had given way as a result of the high obstruction and produced an oesophago-pleural fistula.

The gangrenous patches on the stomach wall were oversewn and the stomach was returned with some difficulty to the abdominal cavity. The hiatus was again repaired in a similar fashion to that used after the Heller's operation. The fistula in the oesophagus was too oedematous and friable to be repaired. The chest was again closed with basal and apical tubes. Through a left upper paramedian incision the abdominal cavity was then opened, a jejunostomy performed, and the anterior wall of the stomach anchored to the anterior abdominal wall.

The patient's recovery after this operation was surprisingly good and was complicated only by some troublesome parotitis. She was fed exclusively through the jejunostomy for 10 days but the tube was removed after 14 days when normal feeding was re-established. The pleural drainage tubes were removed after 48 


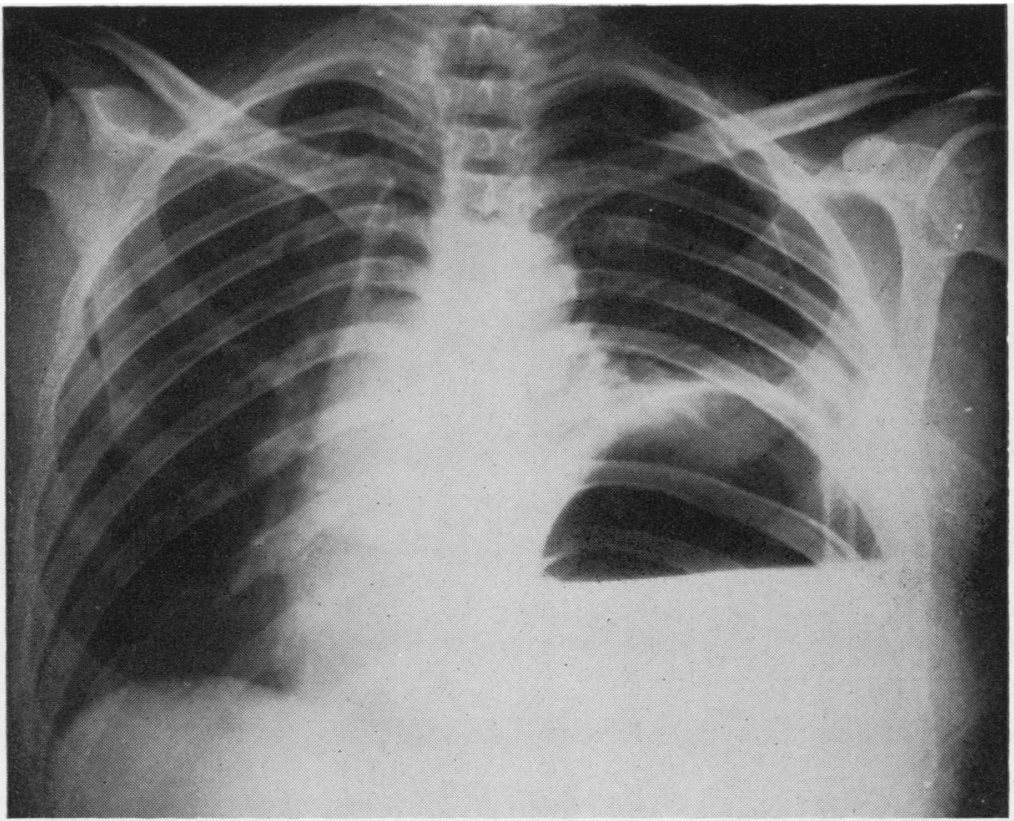

FIG. 3. A postero-anterior radiograph of the chest on the third post-operative day showing considerable enlargement of the fluid level.

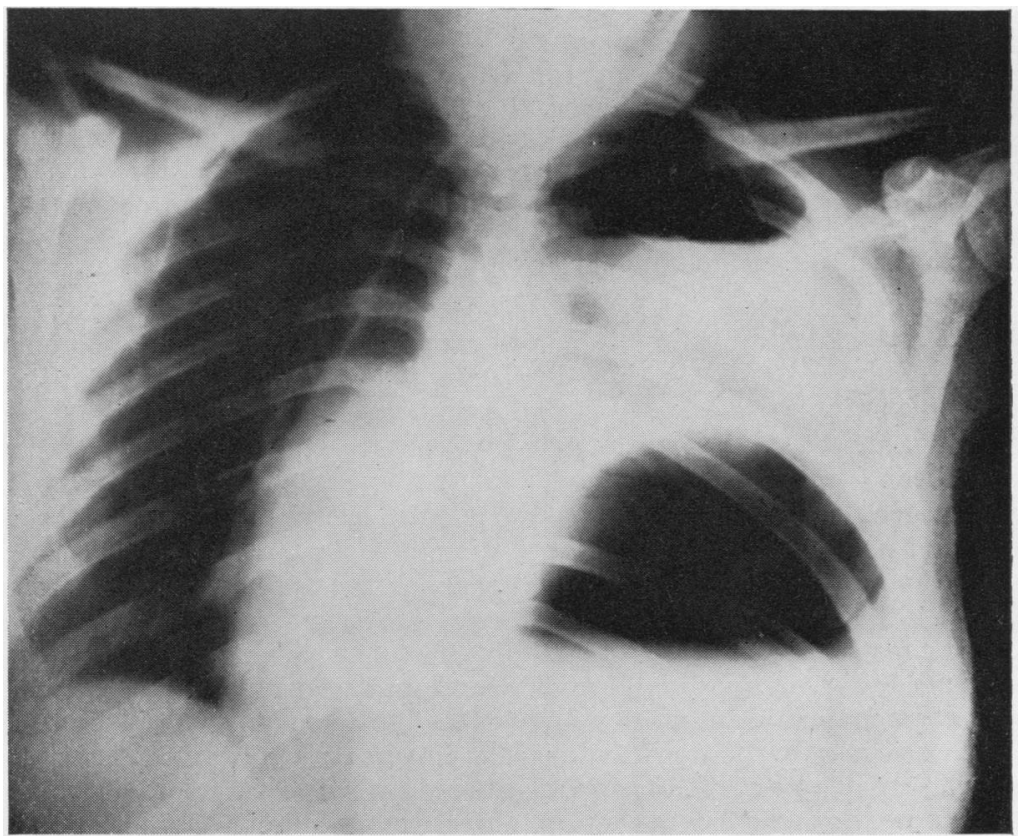

FIG. 4. A postero-anterior rudiograph of the chest on the fourth post-operative day showing a second fluid level at the apex and a large pleural effusion. 

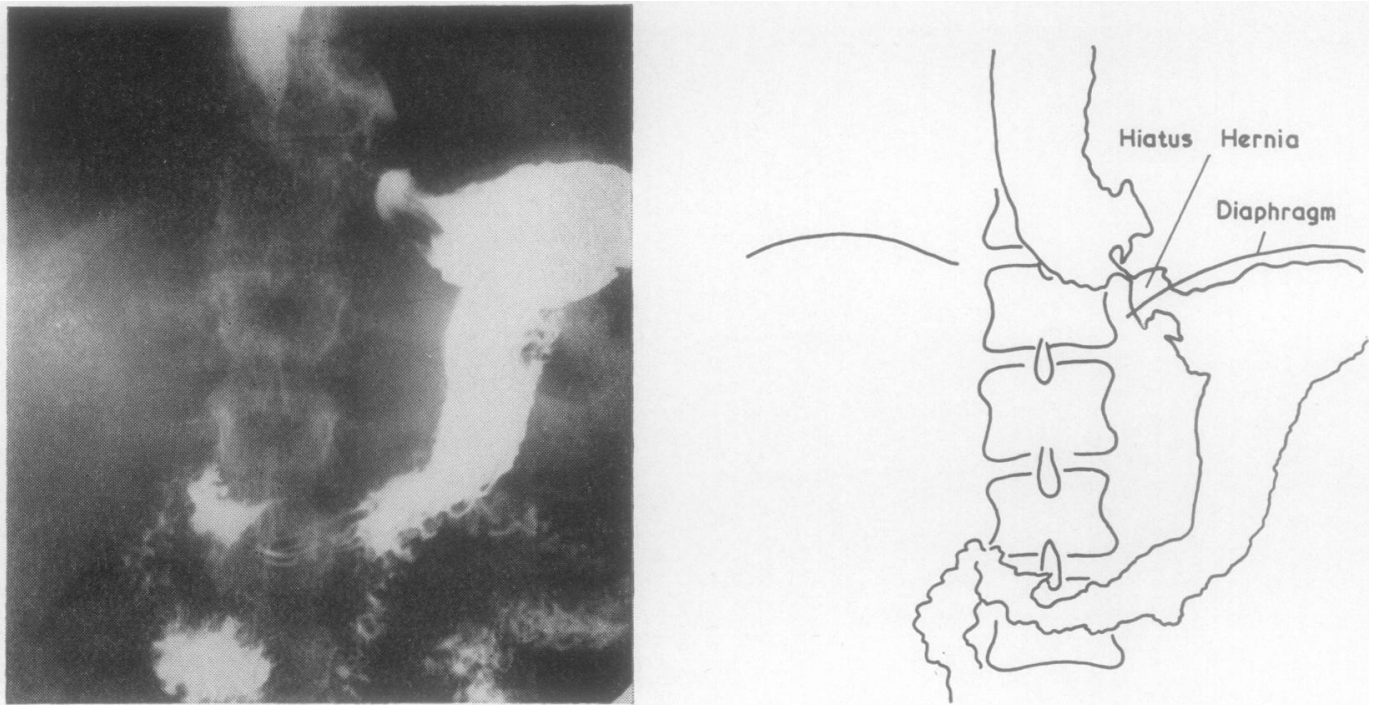

FIG. 5. A routine barium study six months post-operatively showing a hiatus hernia.

hours and the lung remained fully re-expanded with no residual empyema. The patient was finally discharged home on 15 December 1960 on a full diet with no difficulty in swallowing.

When seen six months later she still had no difficulty in swallowing and had put on $42 \mathrm{lb}$. (19.05 kg.) in weight, but on direct questioning she admitted to having occasional heartburn. Routine radiography then showed a small hiatus hernia with free reflux into a still rather dilated oesophagus (Fig. 5). One year after her operation she is still having some heartburn though not enough to cause her great discomfort, but there has been no recurrence of her dysphagia and her weight has been maintained.

\section{DISCUSSION}

The relation of achalasia of the cardia and hiatus hernia has already been the subject of much discussion. Their coexistence is difficult to understand as the mechanisms of their production appear to be almost diametrically opposed. This is borne out by Kay (1953), who has studied the inferior oesophageal constriction in relation to various disorders of the lower oesophagus. These findings have not prevented many authorities on oesophageal surgery, as mentioned by Ravitch (1958), from believing that some of the poor results after Heller's operation can be explained by a hiatus hernia which is not necessarily the result of the operation but is coexistent preoperatively.
Reported cases of coexistent achalasia of the cardia and hiatus hernia, such as that of Euphrat and Delmonico (1957), are however still rare.

Hiatus hernia after Heller's operation is reported much more fully and is much more readily understood. Barrett's and Franklin's (1949) belief that Heller's operation does not produce incompetence of the cardia and oesophagitis is well founded in a direct sense but the fact remains that incompetence and oesophagitis are certainly produced in a proportion of patients after Heller's operation by the indirect effect of a hiatus hernia. Thus, while there is good evidence that Heller's operation is more satisfactory than alternative operative procedures, the complete absence of regurgitation that follows it is not a valid argument in its favour.

Frobese et al. (1961) have reported four cases of hiatus hernia in patients who have undergone Heller's operation. Two of these patients were found to have a hiatus hernia on radiological examination because of heartburn while the remaining two, though found to have hiatus hernia on routine radiological examination, also developed symptoms of oesophagitis at a later date. Though one of the hiatus herniae reported by Frobese and his associates is described as irreducible when repaired, the herniae had given rise to no more serious complication than symptoms of regurgitation.

The hiatus hernia after Heller's operation reported here developed in the immediate post- 
operative period, was sudden and dramatic in its presentation, and was extremely dangerous in its effects. Though there was a history of heartburn prior to the onset of gross difficulty in swallowing, no hiatus hernia was seen on the barium studies pre-operatively and there was nothing to suggest at operation that the hiatus was in any way abnormal. The hiatus was carefully repaired after the Heller's operation. This did not prevent the hiatus hernia, however, and the case may be compared with one of those reported by Frobese and his associates (1961) where the hiatus was also repaired through the chest. The incidence of hiatus hernia is said to be greater where the Heller's operation is performed through the abdomen, but it is obvious that the prevention of a hiatus hernia cannot be completely guaranteed even when the hiatus is deliberately repaired under good vision via the pleural cavity. This is particularly true when one considers how small the hiatus need be, as witnessed in the case presented here, where the hiatus was so small and tight as to produce gangrenous patches on the stomach wall and complete intestinal obstruction.

The part that the negative pressure, applied to the drainage tubes, played in the production of the hiatus hernia is difficult to assess. During the three hours or so when the high negative pressure was inadvertently applied to the drainage tubes the patient did not complain in any way, but the next radiological examination some hours later showed the small fluid level (Fig. 2). It was not until a further 24 hours had elapsed that the patient complained of sudden pain in the chest and another 24 hours again before great enlargement in the fluid level was evident on chest radiography, so that obviously the high pressure was not responsible for the complete development of the hiatus hernia. It may well have been that the short period of high negative pressure drew a small portion of the fundus of the stomach through the hiatus to give the small fluid level seen on chest radiography and to begin the process which the combination of positive intra-abdominal pressure and negative intrathoracic pressure carried on to produce the large hiatus hernia found at operation.

As more and more attention is being drawn to hiatus hernia complicating Heller's operation, more care will be taken in reconstruction of the hiatus. It seems that a certain proportion of cases are still likely to occur as the patient reported here still has a small hiatus hernia even after two deliberate attempts at adequate closure of the hiatus through the chest, together with anchoring of the stomach to the anterior abdominal wall.

In the post-operative management of this patient the fluid levels in the early radiographic examinations were thought to be pleural spaces. In retrospect the post-operative management would have been much improved, and the patient's general condition would not have been allowed to degenerate so badly, if the possibility of herniation of the stomach had been considered. Chest radiography after a Dionosil swallow, carried out in the early stages, would probably have given this diagnosis and the corrective procedures could have been instituted at an earlier stage.

\section{SUMMARY}

A case of strangulated para-oesophageal hiatus hernia arising as an immediate post-operative complication of a Heller's operation for achalasia of the cardia is presented.

The relation of achalasia of the cardia and hiatus hernia is discussed.

The part played by high negative intrapleural pressures in the post-operative phase is also discussed.

It is shown that two reconstructions of the hiatus did not guarantee against the recurrence of a small hiatus hernia.

\section{REFERENCES}

Barrett, N. R., and Franklin, R. H. (1949). Brit. J. Surg., 37, 194. Euphrat, E. J., and Delmonico, J. E., Jr. (1957). J. thorac. Surg., 34, 395.

Frobese A. S., Stein, G. N., and Hawthorne, H. R. (1961). Surgery $49,599$.

Kay, E. B. (1953). J. thorac. Surg., 25, 1.

Ravitch, M. M. (1958). Ibid., 36, 460. 